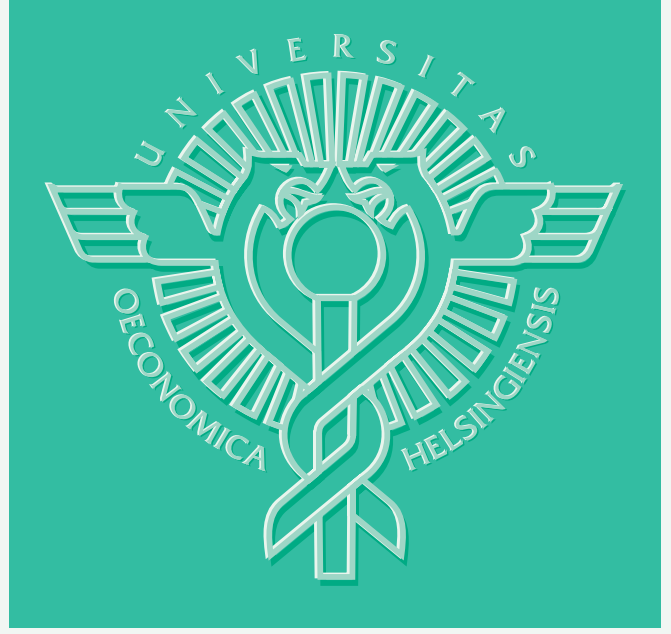

Pekka J. Korhonen - Pyry-Antti Siitari

\title{
A DIMENSIONAL DECOMPOSITION APPROACH TO IDENTIFYING EFFICIENT UNITS IN LARGE-SCALE DEA MODELS
}


Pekka J. Korhonen - Pyry-Antti Siitari

\title{
A DIMENSIONAL DECOMPOSITION APPROACH TO IDENTIFYING EFFICIENT UNITS IN LARGE-SCALE DEA MODELS
}

\author{
Management Science \\ March \\ 2007
}


HELSINGIN KAUPPAKORKEAKOULU

HELSINKI SCHOOL OF ECONOMICS

PL 1210

FI-00101 HELSINKI

FINLAND

(C) Pekka J. Korhonen, Pyry-Antti Siitari and

Helsinki School of Economics

ISSN 1235-5674

(Electronic working paper)

ISBN 978-952-488-124-1

Helsinki School of Economics -

HSE Print 2007 


\title{
A DIMENSIONAL DECOMPOSITION APPROACH TO IDENTIFYING EFFICIENT UNITS IN LARGE-SCALE DEA MODELS
}

\author{
Pekka J. Korhonen \& Pyry-Antti Siitari
}

(March 2007)

\author{
Helsinki School of Economics \\ Department of Business Technology \\ P.O. Box 1210, 00101 Helsinki, FinLAND \\ Tel. +358-9-431 31 \\ Fax. +358-9-43138535 \\ E-mail: Pekka.Korhonen@hkkk.fi / Siitari@,hkkk.fi
}

The research was supported by the Academy of Finland.

All rights reserved. This study may not be reproduced in whole or in part without the authors' permission.

Acknowledgements: We would like to thank Prof. Jose Dulá for useful discussions and providing us with the data files we used in our performance tests. We also thank Dr. Victor Podinovski and Ms. Luiza Badin for their useful comments 


\begin{abstract}
In this paper, we propose the use of a dimensional decomposition procedure together with lexicographic parametric programming to reduce computational burden when identifying the efficient decision making units in Data Envelopment Analysis (DEA). The use of lexicographic parametric programming makes it possible to develop an efficient algorithm for the problems with few inputs and outputs. That's why we propose the procedure which first partitions the original problem dimensionally into sub-problems and then identifies the efficient units of the sub-problems. Because those units are a subset of the weakly efficient solutions of the original problem, they are used as an initial approximation for the efficient units of the original problem. The efficiency of the approach is illustrated by numerical results.
\end{abstract}

Keywords: Efficiency Analysis, Data Envelopment Analysis, Decomposition, Computational Aspects

\title{
1 INTRODUCTION
}

Data Envelopment Analysis (DEA) was originally proposed by Charnes, Cooper and Rhodes [1978 and 1979] as a method for evaluating the relative (technical) efficiency of different Decision Making Units (DMUs) essentially performing the same task. Each of the units uses multiple inputs to produce multiple outputs. The units are assumed to operate under similar conditions. The DEA is value-free in the sense that efficiency evaluation is based on the data available without taking into account the decision maker's (DM) preferences.

Based on information about existing data on the input/output-values of the units and some preliminary assumptions, a so-called production possibility set (PPS) is specified. The production possibility set consists of all possible input/output combinations. A specific part of the production possibility set is called an efficient frontier (surface). The input/output - values on the efficient frontier have a property that none of the inputs or outputs can be improved without worsening at least one input or output. If a DMU lies on the efficient frontier, it is referred to as an efficient unit, otherwise it is considered inefficient. DEA also provides efficiency scores and reference set for inefficient DMUs. The efficiency scores represent a degree of inefficiency of the DMUs. The reference set for inefficient units consists of a set of efficient units and determines a virtual target unit on the efficient frontier.

The target unit is found in DEA by projecting an inefficient DMU radially ${ }^{1}$ to the efficient surface. To check the efficiency of a unit and to find the reference set and the efficiency score for inefficient units requires the solving of an LP-model. The straightforward method is to solve iteratively an LP-model for each unit separately. The optimal basis of the previous iteration is not valid for the next iteration as such, because at each iteration, the right-hand side vector and one column in the coefficient matrix has to be updated.

\footnotetext{
${ }^{1}$ Term "radial" in the traditional DEA-literature means that an efficient frontier is tried to reach by increasing the values of the current outputs or decreasing the values of the current inputs.
} 
When the number of the units is tens or even hundreds of thousands, computationally more efficient methods are needed. Such problems appear when, for example, the efficiency analysis is made at an individual level. In these kinds of problems, the straightforward approach to formulate an LP-model for each unit with an unknown status does not work. It is too time-consuming. Fortunately, the structure of the DEA-model makes it possible to develop special techniques for large-scale problems.

There are not very many authors who have studied computational problems in DEA. One of those exceptions is Ali [1993, 1994], who proposed an idea to restrict the basis entry. The basis always consists of a set of existing efficient units. When a unit is diagnosed inefficient, the corresponding column can be dropped from the set of potential basic vectors. Moreover, Dulá and Helgason [1996] proposed solving the problem in two phases. In phase I, the extreme point solutions of the polytope consisting of all units in the data set are defined. The efficiency scores of the other vectors are computed in phase II by using the minimal set of potential basic vectors, i.e. efficient units. The idea was further developed in the paper by Dulá et al. [1997]. The more recent developments by Dulá and his associates are presented in Dulá and López [2002].

Because the computing time as the function of the units increases more than linearly, Barr and Durchholz [1997] proposed the partition of the set of the units. The efficient units are first identified in a small data set, and then the union of those units is used to build a set of potential basic vectors for the original problem. The union consists of all efficient units, but usually also inefficient units. The idea leads to computationally efficient algorithms, because the savings in computing time are remarkable in comparison to solving the original problem in one phase.

In our previous paper (Korhonen and Siitari [2007]), we used lexicographic parametric programming (Korhonen and Halme [1996]) to traverse from unit to unit along the efficient frontier. On the way, it was possible to recognize a set of units efficient or inefficient. The procedure is computationally efficient, when the number of inputs and outputs is small.

In this paper, we propose a dimensional decomposition to improve the performance of the procedure by Korhonen and Siitari [2007]. An original set is divided dimensionally into subsets, and the efficient units are identified in each subset. Those units are the weakly efficient units of the original problem. However, they provide a good initial approximation for the set of all efficient units of the original problem. The size of that set is usually much smaller than the number of the original units. The set is supplemented during the solution procedure with the units which are diagnosed super-efficient (Andersen and Petersen [1993]). Finally, the efficient units of the original problem are recognized from this supplemented set.

The paper is given in five sections. In the next section, the necessary theoretical questions are discussed. Section 3 represents the algorithm developed in this paper. Computational results are given and discussed in section 4. Section 5 concludes the paper with some remarks. 


\section{THEORETICAL CONSIDERATIONS}

\subsection{Preliminary Considerations}

Consider a production technology, where $m$ inputs are needed to produce $p$ outputs. We denote inputs by $\boldsymbol{x} \in \mathfrak{R}_{+}^{m}$ and outputs by $\boldsymbol{y} \in \mathfrak{R}_{+}^{p}$. We define the production possibility set (PPS):

$$
\mathrm{P}^{0}=\{(\boldsymbol{y}, \boldsymbol{x}) \mid \boldsymbol{y} \text { can be produced from } \boldsymbol{x}\} \in \mathfrak{R}_{+}^{p+m}
$$

which consists of all feasible inputs and outputs in the general sense that the inputs are capable of producing the outputs. We assume that both inputs and outputs are freely disposable. As usually, we assume that in outputs more is better and in inputs less is better.

In practice, set $\mathrm{P}^{0}$ is unknown. To approximate $\mathrm{P}^{0}$, we usually gather sample information about the existing units, set up some assumptions, and define a set $\mathrm{P}$, which is assumed to be a subset of $\mathrm{P}^{0}$.

Now we are ready to define some efficiency concepts for the elements of the production possibility set $\mathrm{P}$.

Definition 1. A point $\left(y^{*}, x^{*}\right) \in \mathrm{P}$ is efficient in set $\mathrm{P}$ iff (if and only if) there does not exist another $(y, x) \in \mathrm{P}$ such that $\boldsymbol{y} \geq y^{*}, x \leq x^{*}$ and $(y, x) \neq\left(y^{*}, x^{*}\right)$.

Definition 2. A point $\left(y^{*}, x^{*}\right) \in \mathrm{P}$ is weakly efficient in set $\mathrm{P}$ iff there does not exist another $(\boldsymbol{y}, \boldsymbol{x}) \in \mathrm{P}$ such that $\boldsymbol{y}>\boldsymbol{y}^{*}$ and $\boldsymbol{x}<\boldsymbol{x}^{*}$.

Definition 3. A point $\left(y^{*}, x^{*}\right) \in \mathrm{P}^{0}-\mathrm{P}$ is super-efficient with respect to set $\mathrm{P}$ iff there does not exist $(\boldsymbol{y}, \boldsymbol{x}) \in \mathrm{P}$ such that $\boldsymbol{y} \geq \boldsymbol{y}^{*}, \boldsymbol{x} \leq \boldsymbol{x}^{*}$.

To simplify notations in the following considerations, we define set Q:

$$
(\boldsymbol{y}, \boldsymbol{x}) \in \mathrm{P} \Leftrightarrow(\boldsymbol{y},-\boldsymbol{x}) \in \mathrm{Q},
$$

and a dimensional chip of $\mathrm{Q}: \mathrm{Q}_{\mathrm{B}} \in \mathfrak{R}_{+}^{h}, h<p+m$

$$
\mathrm{Q}_{\mathrm{B}}=\left\{q_{B} \mid q=\left(\begin{array}{c}
q_{A} \\
q_{B}
\end{array}\right) \in \mathrm{Q}\right\} \text {. }
$$

Theorem 1. If $q_{B}^{*} \in \mathrm{Q}_{\mathrm{B}}$ is weakly efficient in $\mathrm{Q}_{\mathrm{B}}$, then all $q^{*}=\left(\begin{array}{c}q_{A}^{*} \\ q_{B}^{*}\end{array}\right) \in \mathrm{Q}$ are weakly efficient in Q.

Proof: Assume $q^{*}=\left(\begin{array}{c}q_{A}^{*} \\ q_{B}^{*}\end{array}\right) \in \mathrm{Q}$ is not weakly efficient. Then there is another $q=\left(\begin{array}{l}q_{A} \\ q_{B}\end{array}\right) \in \mathrm{Q}$ such that $q>q^{*}$. However, it leads to the contradiction with the assumption that $q_{B}^{*} \in \mathrm{Q}_{\mathrm{B}}$ is weakly efficient in $\mathrm{Q}_{\mathrm{B}}$, because there cannot exist another $q_{B} \in \mathrm{Q}_{\mathrm{B}}$ such that $q_{B}>q_{B}^{*} \square$ 
Remark 1. If point $q_{B}^{*}$ is efficient in $\mathrm{Q}_{\mathrm{B}}, q^{*}$ is weakly efficient in $\mathrm{Q}$, but not necessarily efficient.

Remark 2. If point $q^{*}$ is (weakly) efficient in $\mathrm{Q}$, then point $q_{B}^{*}$ may be (weakly) efficient or inefficient in $\mathrm{Q}_{\mathrm{B}}$.

\subsection{Basic Data Envelopment Models}

Assume we have $n$ DMUs each consuming $m$ inputs $(m \neq 0)$, and producing $p$ outputs $(p \neq 0)$. Information can be presented by two matrices. Let $\mathbf{X}$ be an $(m \times n)$ matrix and $\mathbf{Y}$ be a $(p \times n)$ - matrix consisting of non-negative elements, containing observed input and output measures for the DMUs, respectively. We denote by $\boldsymbol{x}_{j}$ (the $j$ th column of $\mathbf{X}$ ) the vector of inputs consumed by $\mathrm{DMU}_{j}$, and by $x_{i j}$ the quantity of input $i$ consumed by $\mathrm{DMU}_{j}$. A similar notation is used for outputs. We further assume that $\boldsymbol{x}_{j} \neq \boldsymbol{0}$ and $\boldsymbol{y}_{j} \neq \boldsymbol{0}, j=1,2, \ldots, n$, and for simplicity, we assume that there are no duplicates in the data set. Furthermore, we denote $1=[1, \ldots, 1]^{\mathrm{T}}$.

Using the existing data set, we define the (practical) production possibility set as a set $\mathrm{P}=\{(\boldsymbol{y}, \boldsymbol{x}) \mid \boldsymbol{x} \geq \mathbf{X} \boldsymbol{\lambda}, \boldsymbol{y} \leq \mathbf{Y} \lambda, \lambda \in \Lambda\}$, where the definition of $\Lambda$ depends on the assumptions made about the form of set $\mathrm{P}$.

Data Envelopment Analysis models can be interpreted as tools to check which existing or virtual units are on the efficient frontier of P. The traditional CCR-models, as introduced by Charnes et al. [1978, 1979] are originally presented as fractional linear programs which can easily be formulated and solved as linear programs. Those models are so-called constant returns to scale models. Later Banker, Charnes and Cooper [1984] developed the so-called BCC models with variable returns to scale ${ }^{2}$. The CCR and BCC models are the basic model types in DEA. Those basic models can be presented in a primal or dual form. Which one is primal or dual varies. It is more recommendable to call them multiplier and envelopment models accordingly. The multiplier model provides information on the weights of inputs and outputs which are interpreted as prices in many applications. Instead, the envelopment models provide the user with information on the lacks of outputs and the surplus of inputs of a unit. Moreover, the envelopment model characterizes the reference set for inefficient units.

The models can be further classified as input-oriented or output-oriented models. The model is input-oriented, when the output levels are given, and the inputs are tried to reduce radially in the production feasibility set. In the output-oriented models, the role of inputs and outputs are changed with the exception that the outputs are tried to increase. Without loss of generality, we may also consider a DEA-model by using a general directional vector $\boldsymbol{w}=\left(\begin{array}{c}\boldsymbol{w}^{y} \\ \boldsymbol{w}^{x}\end{array}\right) \geq \boldsymbol{0}, \boldsymbol{w} \neq \boldsymbol{0}$ (discussion on directional distance functions, see Chambers et al. [1998]). Halme et al. [1999] called the model a general combined model. Input- and output-oriented models are the special cases of that model.

Consider the following lexicographic formulation of the general DEA-model in the so-called envelopment form (Korhonen and Siitari [2007]):

\footnotetext{
${ }^{2}$ Actually, the BCC model was first proposed by Afriat [1972, p.581], in a case of one output. The general version of the envelopment BCC model was independently also proposed, implemented and solved by Färe, Grosskopf and Logan [1983].
} 


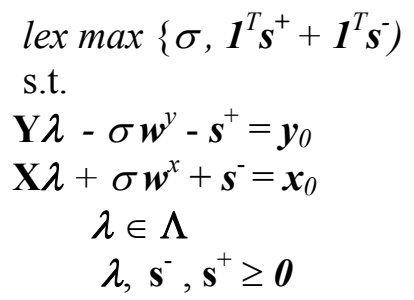

where $\boldsymbol{x}_{0}$ is the input-vector and $\boldsymbol{y}_{0}$ is the output-vector of a DMU under consideration and

$$
\Lambda=\left\{\begin{array}{l}
\left\{\boldsymbol{\lambda} \mid \boldsymbol{1}^{\prime} \boldsymbol{\lambda}=1, \boldsymbol{\lambda} \geq \boldsymbol{0}\right\} \text { for variable returns to scale model (Banker et al. [1984]) } \\
\left\{\boldsymbol{\lambda} \mid \boldsymbol{1}^{\prime} \boldsymbol{\lambda} \leq 1, \boldsymbol{\lambda} \geq \boldsymbol{0}\right\} \text { for non-increasing returns to scale model } \\
\left\{\boldsymbol{\lambda} \mid \boldsymbol{1}^{\prime} \boldsymbol{\lambda} \geq 1, \boldsymbol{\lambda} \geq \boldsymbol{0}\right\} \text { for non-decreasing returns to scale model } \\
\{\boldsymbol{\lambda} \mid \boldsymbol{\lambda} \geq \boldsymbol{0}\} \text { for constant returns to scale model (Charnes } \text { et al. [1978]). }
\end{array}\right.
$$

Notation "lex max" refers to a lexicographic maximization problem. It means that we first solve (2.4) using $\sigma$ as an objective function. In case, the optimal solution $\sigma^{*}$ is not unique, we add the constraint $\sigma=\sigma^{*}$ into the model (2.4) and solve it by using $\boldsymbol{I}^{T} \boldsymbol{s}^{+}+\boldsymbol{I}^{T} \boldsymbol{s}^{-}$as the objective function.

The first three constraints for $\lambda$ specifies one of the BCC - models, and the last one specifies the CCR-model. In the combined model, $\boldsymbol{w}^{y}=\boldsymbol{y}_{0}$ and $\boldsymbol{w}^{x}=\boldsymbol{x}_{0}$. In the inputoriented model $\boldsymbol{w}^{y}=\boldsymbol{0}$ and $\boldsymbol{w}^{x}=\boldsymbol{x}_{0}$, and in the output-oriented model $\boldsymbol{w}^{x}=\boldsymbol{0}$ and $\boldsymbol{w}^{y}=$ $\boldsymbol{y}_{0}$. The value of $\sigma$-called an inefficiency score - at the optimum is denoted by $\sigma^{*}$. A DMU is efficient iff the optimal value $\sigma^{*}$ and all slack variables $\boldsymbol{s}^{-}, \boldsymbol{s}^{+}$equal zero. Otherwise, the DMU is inefficient (Charnes et al. 1994). For weakly-efficient solutions $\sigma^{*}=0$ but $\left(\boldsymbol{1}^{T} \boldsymbol{s}^{+}+\boldsymbol{1}^{T} \boldsymbol{s}^{-}\right)>0$.

\subsection{Problem Decomposition}

Let $\mathrm{N}$ be the index set of $n$ DMUs $(\mathrm{N}=\{1,2, \ldots, n\})$ in the original problem. Let I be the index set of the rows of $\mathrm{X}(\mathrm{I}=\{1,2, \ldots, m\})$ and let $\mathrm{O}$ be the index set of the rows of $\mathrm{Y}(\mathrm{O}=\{1,2, \ldots, p\})$. By dimensional decomposition we mean that the set of input variables $\mathrm{I}$ and the set of output variables $\mathrm{O}$ are divided into $q$ subsets: $\mathrm{I}=\mathrm{I}_{l} \cup$ $\mathrm{I}_{2} \cup \ldots \cup \mathrm{I}_{q}, \mathrm{I}_{i} \neq \varnothing$, and $\mathrm{O}=\mathrm{O}_{l} \cup \mathrm{O}_{2} \cup \ldots \cup \mathrm{O}_{q}, \mathrm{O}_{i} \neq \varnothing$. The sets are not necessarily disjoint. Each pair of sets $\left(\mathrm{I}_{i}, \mathrm{O}_{i}\right)$ defines a DEA sub-problem $\mathrm{A}_{i}(i=1,2, \ldots, q)$. Each sub-problem specifies a status for each $\mathrm{DMU}_{j}, j=1,2, \ldots, n$. The set of efficient DMUs in sub-problem $\mathrm{A}_{i}$ is denoted by $\mathrm{K}_{i}^{\mathrm{E}}$, the set of only weakly efficient DMUs by $\mathrm{K}_{i}^{\mathrm{W}}$ and the set of super-efficient DMUs by $\mathrm{K}_{i}^{\mathrm{S}}$. We drop the subscript, when we refer to the original problem.

In the proposed algorithm, each $\mathrm{DMU}_{j}, j \in \mathrm{N}$ is classified to one sub-problem $\mathrm{A}_{i}(i=$ $1,2, \ldots, q)$. The index set $\mathrm{N}_{i}$ refers to the DMUs belonging to sub-problem $\mathrm{A}_{i}$.

We denote $\mathrm{A}_{r} \subseteq \mathrm{A}_{v}$, if $\mathrm{I}_{r} \subseteq \mathrm{I}_{v}$ and $\mathrm{O}_{r} \subseteq \mathrm{O}_{v}$. In this case, problem $\mathrm{A}_{v}$ has at least all the variables that the problem $\mathrm{A}_{r}$ contains. The problem $\mathrm{A}_{r}$ is a dimensional chip of the problem $\mathrm{A}_{v}$. Each unit in the problem $\mathrm{A}_{r}$ can be interpreted as a vector $\boldsymbol{q}_{B}$ in (2.3). Similarly the production possibility set of $\mathrm{A}_{r}$ relates to the production possibility set 
of $\mathrm{A}_{v}$ as the vector set $\mathrm{Q}_{\mathrm{B}}$ relates to $\mathrm{Q}$ in (2.3). By Theorem 1, if $\mathrm{A}_{r} \subseteq \mathrm{A}_{v}$, then $\mathrm{K}_{r}^{\mathrm{E}} \subseteq$ $\left(\mathrm{K}_{v}{ }^{\mathrm{W}} \cup \mathrm{K}_{v}{ }^{\mathrm{E}}\right)$.

By evaluating dimensionally decomposed problems we can expect to find several units that are efficient also in the original (full dimensional) problem. Using these units as potential basic variables we can classify all the other DMUs either inefficient or super-efficient with respect to our new frontier.

\section{THE ALGORITHM}

\subsection{Decomposition algorithm}

The problem with $m+p>1$ variables can be decomposed many ways. It is important to choose the decomposition in such a way that we get "efficiency information" as much as possible. If e.g. $\Lambda=\{\boldsymbol{\lambda} \mid \boldsymbol{\lambda} \geq \boldsymbol{0}\}$, then in case we choose all variables to be maximized or to be minimized, all units are inefficient. This kind of decomposition does not give us much useful information. In advance, it is hard to say which decomposition is best. If in each chip there is at least one input to be minimized and one output to be maximized, then each sub-problem has at least one efficient unit. In addition, it is reasonable to minimize the overlapping of the variables between the sub-problems. Motivation to decompose the problem dimensionally is to recognize different efficient DMUs of the original problem. That's why it is justified to try to have as diverse set of variables in each problem as possible.

In this paper we have used the following algorithm to divide the set of input variables $\mathrm{I}$ and the set of output variables $\mathrm{O}$ into $q$ subsets $\mathrm{I}_{1}, \mathrm{I}_{2}, \ldots, \mathrm{I}_{q}$ and $\mathrm{O}_{1}$, $\mathrm{O}_{2}, \ldots, \mathrm{O}_{q}$.

\section{Step 0: Initialization}

Select block-quantity $q(1 \leq q \leq \max (m, p))$ which determines how many subproblems will be created. The value for $q$ will be determined empirically. Define two temporary index sets $\mathrm{I}_{t}$ and $\mathrm{O}_{t}$.

Step 1: Decomposing input variables

Set $\mathrm{I}_{t}:=\mathrm{I}$. Define $k:=1$.

Set allInputsUsed $:=$ false and allSubsetsUsed $:=$ false.

\section{Step 1a:}

Step 1b:

Select the first variable $i$ from the index set $\mathrm{I}_{t}$. Redefine $\mathrm{I}_{k}:=\mathrm{I}_{k}+i$, $\mathrm{I}_{t}:=\mathrm{I}_{t}-i$ and $k:=k+1$.

If $\left(\mathrm{I}_{t}=\varnothing\right)$ (if all input variables have been used at least once) Redefine $\mathrm{I}_{t}:=\mathrm{I}$. Set allinputsUsed $:=$ true.

If $(\mathrm{k}=\mathrm{q})$ (if all subsets have at least one input) Redefine $k:=1$. Set allSubsetsUsed $:=$ true.

If $($ allinputsUsed $=$ true $)$ and $($ allSubsetsUsed $=$ true $)$ Go to Step 2.

Else 
Go to Step 1a.

Step 2: Decomposing output variables

Set $\mathrm{O}_{t}:=\mathrm{O}$. Define $k:=q$.

Set allOutputsUsed $:=$ false and allSubsetsUsed $:=$ false.

Step 2a:

Select the first variable $o$ from the index set $\mathrm{O}_{t}$. Redefine $\mathrm{O}_{k}:=\mathrm{O}_{k}+$ $o$,

Step 2b:

$\mathrm{O}_{t}:=\mathrm{O}_{t}-o$ and $k:=k-1$.

If $\left(\mathrm{O}_{t}=\varnothing\right)$ (if all output variables have been used at least once)

Redefine $\mathrm{O}_{t}:=\mathrm{O}$. Set allOutputsUsed $:=$ true.

If $(\mathrm{k}=0)$ (if all subsets have at least one output)

Redefine $k:=q$. Set allSubsetsUsed $:=$ true.

If $($ allOutputsUsed $=$ true $)$ and $($ allSubsetsUsed $=$ true $)$

Stop.

Else

Go to Step 2a.

\subsection{Classification algorithm}

In this section we describe the proposed algorithm for solving the decomposed problem.

Step 0: Initialization

Divide the set of input variables I and the set of output variables $\mathrm{O}$ into $q$ subsets $\mathrm{I}_{1}$, $\mathrm{I}_{2}, \ldots, \mathrm{I}_{q}$ and $\mathrm{O}_{1}, \mathrm{O}_{2}, \ldots, \mathrm{O}_{q}$ as described in the decomposition algorithm. For each pair of subsets, form a new dimensionally decomposed DEA sub-problem $\mathrm{A}_{i}(i=1,2, \ldots$, $q)$.

Step 1: Dimensional Decomposition

Solve each of the sub-problems $\mathrm{A}_{i}$. Get the union of efficient units $\mathrm{K}^{\mathrm{E}^{*}}$ in each subproblem $\mathrm{K}_{1}{ }^{\mathrm{E}} \cup \mathrm{K}_{2}{ }^{\mathrm{E}} \cup \ldots \cup \mathrm{K}_{q}{ }^{\mathrm{E}}=\mathrm{K}^{\mathrm{E}^{*}}$

Step 2: Reduction of Number of Potentially Efficient Units

In the set $\mathrm{N}-\mathrm{K}^{\mathrm{E}^{*}}$, identify inefficient units using all inputs and outputs. Use only the units $\mathrm{DMU}_{i}, i \in \mathrm{K}^{\mathrm{E}^{*}}$ as basic units in (2.4). Define a new index set $\mathrm{K}^{\text {super }}$ that includes the set $\mathrm{K}^{\mathrm{E}^{*}}$ and the units that were found either efficient or super-efficient.

Step 3: Recognition of Efficient Units

In the set $\mathrm{K}^{\text {super }}$, identify inefficient units using all variables. The units that are efficient in this problem form the set of efficient units in the original problem.

\section{COMPUTATIONAL RESULTS}

We tested our decomposition procedure using simulated problems, which we received from Prof. Jose Dulá. He has also used these problems in his own tests. The parameters of the problems are the number of units $n$, the number of inputs/outputs 
$m+p$, and the density of the efficient units $d$. The number of units we used was 5000, $10000,15000,20000,25000$, and 50000. The number of inputs/outputs was 20 . We classified the models into three density categories in such a way that the average densities in each class were $6.4 \%, 10.0 \%$, and $22.9 \%$. The computing times are reported using five different block-quantities $q$. The problems are decomposed dimensionally into two $(q=2)$, three $(q=3)$, four $(q=4)$ and five $(q=5)$ subproblems. Computing times without decomposition $(q=1)$ are also reported. The tests are run with a PC-computer with one $2.4 \mathrm{GHz}$ processor. The DEA-model used is CCR.

The test results are reported in Tables 1-4 in each combination of the parameters. In Table 1, we have reported the computing times when the number of sub-problems is two $(q=2)$. In Table 2 the number of sub-problems is three $(q=3)$ and in Tables 3 and 4 the number of sub-problems is four $(\mathrm{q}=4)$ and five $(q=5)$ respectively. Tables 1-4 represent the computing times in each steps together with the number of efficient (Step 1 and Step 3) or super-efficient (Step 2) units found in each step. Column No Part refers to the time (in seconds) needed to solve the problem without decomposition $(q=1)$. Step $1 /$ Time reports the time required to solve the subproblems. Column Step 1 / \# of Alt represents the number of efficient units found in sub-problems. Column Step 2 / Time states the time needed to calculate the superefficiencies. Step 2 / \# of Alt presents the number of super-efficient units found (together with the units found in Step1). Column Step 3 / Time states the time that is needed to calculate the exact set of efficient units. Step 3 / \# of Alt presents the number of efficient units found in the problem. Column Total / Time reports the total time needed to solve the problem by dimensional decomposition approach. Total / Ratio compares the required total time by decomposition with the time without partition. 
Table 1: Computing Times (s) and the Number of Alternatives when the Problem is Decomposed into Two Parts $(q=2)$.

\begin{tabular}{|c|c|c|c|c|c|c|c|c|c|c|}
\hline \multirow[b]{2}{*}{ Density } & \multirow[b]{2}{*}{ \# of Alt. } & \multirow{2}{*}{$\begin{array}{l}\text { No Part. } \\
\text { Time(s) }\end{array}$} & \multirow{2}{*}{\multicolumn{2}{|c|}{$\begin{array}{c}\text { Step1 } \\
\text { Time(s) [\# of Alt. }\end{array}$}} & \multirow{2}{*}{\multicolumn{2}{|c|}{$\begin{array}{c}\text { Step2 } \\
\text { Time(s) |\# of Alt. }\end{array}$}} & \multicolumn{2}{|c|}{ Step3 } & \multicolumn{2}{|c|}{ Total } \\
\hline & & & & & & & Time(s) & \# of Alt. & Time(s) & Ratio \\
\hline \multirow{6}{*}{$6.4 \%$} & 5000 & 354 & 25 & 229 & 16 & \begin{tabular}{|r|}
733 \\
\end{tabular} & \begin{tabular}{|l|}
5 \\
\end{tabular} & $\begin{aligned} 665 \\
\end{aligned}$ & 46 & \begin{tabular}{|l|}
0.129 \\
\end{tabular} \\
\hline & 10000 & 1686 & 99 & 263 & 41 & 855 & 8 & 806 & 148 & 0.088 \\
\hline & 15000 & 3559 & 239 & 274 & $\overline{72}$ & 956 & 11 & 829 & 322 & 0.091 \\
\hline & 20000 & 8589 & 431 & 295 & 127 & 997 & 14 & 888 & 571 & 0.067 \\
\hline & 25000 & 12342 & 705 & 341 & 172 & 1147 & 16 & 1011 & 893 & 0.072 \\
\hline & 50000 & 51753 & 3851 & 456 & 525 & 1642 & 39 & 1376 & 4415 & 0.085 \\
\hline \multirow{6}{*}{$10.0 \%$} & 5000 & 555 & 38 & 297 & 32 & 600 & 3 & 586 & 73 & 0.132 \\
\hline & 10000 & 2935 & 195 & 430 & 97 & 1089 & 12 & 1038 & 304 & 0.103 \\
\hline & 15000 & 6864 & 778 & 549 & 235 & 1590 & 53 & 1498 & 1066 & 0.155 \\
\hline & 20000 & 11425 & 1781 & 641 & 363 & 2055 & 56 & 1930 & 2200 & 0.193 \\
\hline & 25000 & 19209 & 1978 & 707 & 489 & 2384 & 100 & 2384 & 2567 & 0.134 \\
\hline & 50000 & 106172 & 12776 & 1087 & 1559 & 4956 & 523 & 4607 & 14858 & 0.140 \\
\hline \multirow{6}{*}{$22.9 \%$} & 5000 & 675 & 85 & 509 & 65 & 1253 & 16 & 1209 & 166 & 0.245 \\
\hline & 10000 & 3264 & 383 & 712 & 197 & 2441 & 78 & 2346 & 659 & 0.202 \\
\hline & 15000 & 8053 & 1475 & 952 & 438 & 3590 & 233 & 3447 & 2146 & 0.266 \\
\hline & 20000 & 15756 & 2921 & 1058 & 734 & 4770 & 541 & 4517 & 4196 & 0.266 \\
\hline & 25000 & 25089 & 4911 & 1280 & 1152 & 5921 & 900 & 5596 & 6963 & 0.278 \\
\hline & 50000 & 115585 & 21511 & 1802 & 3073 & 11670 & 4033 & 10783 & 28617 & 0.248 \\
\hline
\end{tabular}

Table 2: Computing Times (s) and the Number of Alternatives when the Problem is Decomposed into Three Parts $(q=3)$.

\begin{tabular}{|c|c|c|c|c|c|c|c|c|c|c|}
\hline \multirow[b]{2}{*}{ Density } & \multirow[b]{2}{*}{ \# of Alt. } & \multirow{2}{*}{$\begin{array}{l}\text { No Part. } \\
\text { Time(s) }\end{array}$} & \multirow{2}{*}{\multicolumn{2}{|c|}{$\begin{array}{c}\text { Step1 } \\
\text { Time(s) } \mid \# \text { of Alt. }\end{array}$}} & \multirow{2}{*}{\multicolumn{2}{|c|}{$\begin{array}{c}\text { Step2 } \\
\text { Time(s) |\# of Alt. }\end{array}$}} & \multicolumn{2}{|c|}{ Step3 } & \multicolumn{2}{|c|}{ Total } \\
\hline & & & & & & & Time(s) & $\#$ of Alt. & Time(s) & Ratio \\
\hline \multirow{6}{*}{$6.4 \%$} & 5000 & 354 & 2 & 115 & 8 & 870 & 8 & 665 & \begin{tabular}{|r|}
17 \\
\end{tabular} & 0.048 \\
\hline & 10000 & 1686 & 3 & 124 & 18 & 1271 & 18 & 806 & 39 & 0.023 \\
\hline & 15000 & 3559 & 12 & 134 & 32 & 1388 & 21 & 829 & 64 & 0.018 \\
\hline & 20000 & 8589 & 13 & 134 & 42 & 1551 & 30 & 888 & 85 & 0.010 \\
\hline & 25000 & 12342 & 21 & 163 & 69 & 1599 & 33 & 1011 & 124 & 0.010 \\
\hline & 50000 & 51753 & 54 & 206 & 174 & 2697 & 123 & 1376 & 351 & 0.007 \\
\hline \multirow{6}{*}{$10.0 \%$} & 5000 & 555 & $\overline{2}$ & 151 & 16 & 721 & 5 & 586 & 23 & 0.041 \\
\hline & 10000 & 2935 & 5 & 167 & 32 & 1301 & 20 & 1038 & 57 & 0.020 \\
\hline & 15000 & 6864 & 17 & 212 & 71 & 1799 & 41 & 1498 & 128 & 0.019 \\
\hline & 20000 & 11425 & 30 & 230 & 107 & 2395 & 77 & 1930 & 215 & 0.019 \\
\hline & 25000 & 19209 & 35 & 257 & 136 & 2952 & 140 & 2384 & 312 & 0.016 \\
\hline & 50000 & 106172 & 103 & 330 & 380 & 5643 & 747 & 4607 & 1231 & 0.012 \\
\hline \multirow{6}{*}{$22.9 \%$} & 5000 & 675 & 3 & 197 & 21 & 1328 & 18 & 1209 & 42 & 0.062 \\
\hline & 10000 & 3264 & 9 & 261 & 63 & 2612 & 94 & 2346 & 166 & 0.051 \\
\hline & 15000 & 8053 & 28 & 293 & 100 & 3858 & 300 & 3447 & 428 & 0.053 \\
\hline & 20000 & 15756 & 51 & 328 & 151 & 5129 & 598 & 4517 & 799 & 0.051 \\
\hline & 25000 & 25089 & 60 & 323 & 184 & 6451 & 1008 & 5596 & 1251 & 0.050 \\
\hline & 50000 & 115585 & 210 & 445 & 562 & 12606 & 4889 & 10783 & 5661 & 0.049 \\
\hline
\end{tabular}


Table 3: Computing Times (s) and the Number of Alternatives when the Problem is Decomposed into Four Parts $(q=4)$.

\begin{tabular}{|c|c|c|c|c|c|c|c|c|c|c|}
\hline \multirow[b]{2}{*}{ Density } & \multirow[b]{2}{*}{ \# of Alt. } & \multirow{2}{*}{$\begin{array}{l}\text { No Part. } \\
\text { Time(s) }\end{array}$} & \multirow{2}{*}{\multicolumn{2}{|c|}{$\begin{array}{c}\text { Step1 } \\
\text { Time(s) [\# of Alt. }\end{array}$}} & \multicolumn{2}{|c|}{ Step2 } & \multicolumn{2}{|c|}{ Step3 } & \multicolumn{2}{|c|}{ Total } \\
\hline & & & & & Time(s) & \# of Alt. & Time(s) & \# of Alt. & Time(s) & Ratio \\
\hline \multirow{6}{*}{$6.4 \%$} & 5000 & 354 & 0 & $\begin{array}{r}70 \\
\end{array}$ & 5 & \begin{tabular}{|l|}
1127 \\
\end{tabular} & \begin{tabular}{r|}
13 \\
\end{tabular} & $\begin{array}{r}665 \\
\end{array}$ & 18 & 0.051 \\
\hline & 10000 & 1686 & 1 & 87 & $\overline{13}$ & 1467 & 24 & 806 & 38 & 0.023 \\
\hline & 15000 & 3559 & 1 & 70 & 16 & 1847 & 42 & 829 & 58 & 0.016 \\
\hline & 20000 & 8589 & 4 & 99 & 30 & 2337 & 72 & 888 & 106 & 0.012 \\
\hline & 25000 & 12342 & 4 & 113 & 44 & 2212 & 71 & 1011 & 120 & 0.010 \\
\hline & 50000 & 51753 & 14 & 112 & 97 & 4496 & 510 & 1376 & 621 & 0.012 \\
\hline \multirow{6}{*}{$10.0 \%$} & 5000 & 555 & 0 & 91 & 8 & 823 & 7 & 586 & 16 & 0.028 \\
\hline & 10000 & 2935 & 2 & 113 & 21 & 1400 & 24 & 1038 & 47 & 0.016 \\
\hline & 15000 & 6864 & 3 & 121 & 38 & 2136 & 68 & 1498 & 109 & 0.016 \\
\hline & 20000 & 11425 & 6 & 135 & 67 & 2815 & 149 & 1930 & 222 & 0.019 \\
\hline & 25000 & 19209 & 6 & 122 & 59 & 4061 & 436 & 2384 & 501 & 0.026 \\
\hline & 50000 & 106172 & 19 & 165 & 171 & 6817 & 1442 & 4607 & 1631 & 0.015 \\
\hline \multirow{6}{*}{$22.9 \%$} & 5000 & 675 & 1 & 121 & 12 & 1420 & 22 & 1209 & 34 & 0.051 \\
\hline & 10000 & 3264 & 2 & 160 & 32 & 2736 & 109 & 2346 & 143 & 0.044 \\
\hline & 15000 & 8053 & 7 & 190 & 65 & 4119 & 424 & 3447 & 496 & 0.062 \\
\hline & 20000 & 15756 & 8 & 161 & $\overline{73}$ & 5584 & 864 & 4517 & 945 & 0.060 \\
\hline & 25000 & 25089 & 13 & 198 & 107 & 6884 & 1420 & 5596 & 1540 & 0.061 \\
\hline & 50000 & 115585 & 31 & 204 & 221 & 13678 & 7190 & 10783 & 7442 & 0.064 \\
\hline
\end{tabular}

Table 4: Computing Times (s) and the Number of Alternatives when the Problem is Decomposed into Five Parts $(q=5)$.

\begin{tabular}{|c|c|c|c|c|c|c|c|c|c|c|}
\hline \multirow[b]{2}{*}{ Density } & \multirow[b]{2}{*}{ \# of Alt. } & \multirow{2}{*}{$\begin{array}{l}\text { No Part. } \\
\text { Time(s) }\end{array}$} & \multicolumn{2}{|c|}{ Step1 } & \multicolumn{2}{|c|}{ Step2 } & \multicolumn{2}{|c|}{ Step3 } & \multicolumn{2}{|c|}{ Total } \\
\hline & & & Time(s) & \# of Alt. & Time(s) & \# of Alt. & Time(s) & \# of Alt. & Time(s) & Ratio \\
\hline \multirow{6}{*}{$6.4 \%$} & 5000 & 354 & 0 & 45 & 3 & 1363 & 17 & \begin{tabular}{|r|}
665 \\
\end{tabular} & 20 & 0.058 \\
\hline & 10000 & 1686 & 0 & 48 & 7 & 2476 & 75 & 806 & 82 & 0.049 \\
\hline & 15000 & 3559 & 0 & 50 & 10 & 2694 & 91 & 829 & 101 & 0.028 \\
\hline & 20000 & 8589 & 1 & 60 & 20 & 3770 & 258 & 888 & 279 & 0.032 \\
\hline & 25000 & 12342 & 1 & 53 & 25 & 3329 & 235 & 1011 & 261 & 0.021 \\
\hline & 50000 & 51753 & 2 & 56 & 52 & 6978 & 1497 & 1376 & 1551 & 0.030 \\
\hline \multirow{6}{*}{$10.0 \%$} & 5000 & 555 & 0 & 54 & 4 & 997 & 12 & 586 & 16 & 0.029 \\
\hline & 10000 & 2935 & 0 & 59 & 10 & 1762 & 44 & 1038 & 54 & 0.018 \\
\hline & 15000 & 6864 & 1 & 68 & 17 & 2623 & 115 & 1498 & 133 & 0.019 \\
\hline & 20000 & 11425 & 1 & 57 & 20 & 3940 & 400 & 1930 & 421 & 0.037 \\
\hline & 25000 & 19209 & 1 & 67 & 29 & 4268 & 493 & 2384 & 522 & 0.027 \\
\hline & 50000 & 106172 & 2 & 74 & 68 & 8162 & 2360 & 4607 & 2430 & 0.023 \\
\hline \multirow{6}{*}{$22.9 \%$} & 5000 & 675 & 0 & 58 & 5 & 1576 & 30 & 1209 & 35 & 0.052 \\
\hline & 10000 & 3264 & 0 & 57 & 10 & 3264 & 207 & 2346 & 217 & 0.067 \\
\hline & 15000 & 8053 & 2 & 67 & 24 & 4618 & 542 & 3447 & 567 & 0.070 \\
\hline & 20000 & 15756 & 1 & 60 & 25 & 6321 & 1165 & 4517 & 1191 & 0.076 \\
\hline & 25000 & 25089 & 1 & 75 & 36 & 7773 & 1991 & 5596 & 2028 & 0.081 \\
\hline & 50000 & 115585 & 2 & 81 & 86 & 15847 & 10900 & 10783 & 10988 & 0.095 \\
\hline
\end{tabular}

Tables 1-4 show that the dimensional decomposition approach decreases computation times dramatically. Computation times were smallest with the blockquantities $q=3$ and $q=4$ in all problems. The difference in computation times between these two block-quantities is generally small. In the biggest problems with 50000 units the block-quantity $q=3$ was somewhat faster. We can also see from the Tables 1-4 that the smaller the block-quantity the more time is spent in Step 1 and Step 2 and the more potentially efficient units are found in Step 1. On the other hand, the smaller the block-quantity the smaller the number of super-efficient units found in 
Step 2 and the less time is spent in the Step 3 to calculate the exact set of efficient units.

The computing times are illustrated in Figures 1-3 in each efficiency category using each five block-quantities. In Figure 1, the average efficiency density is $6.4 \%$. In Figure 2 the average efficiency density is $10.0 \%$ and in Figure 3 it is $22.9 \%$.

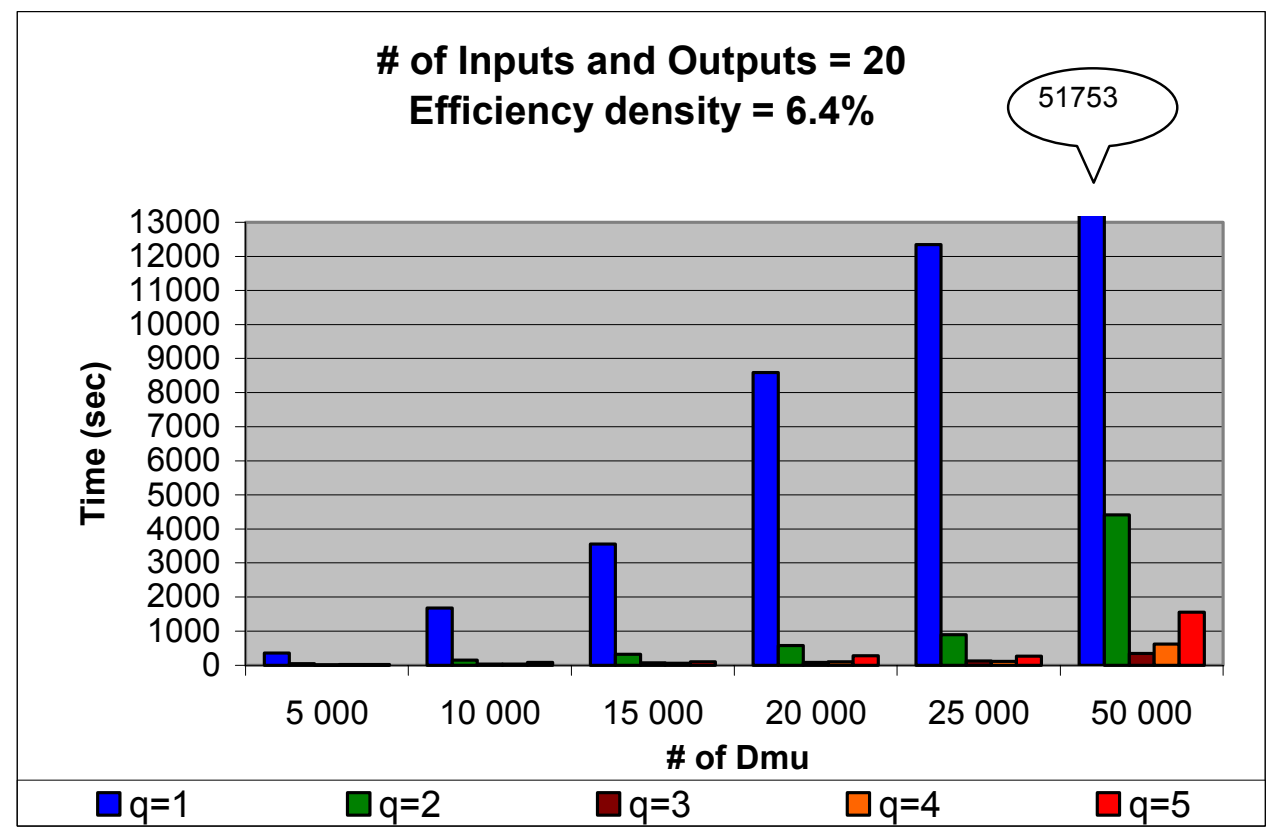

Figure 1: Computing Times (s) with Average Efficiency Density 6.4\%

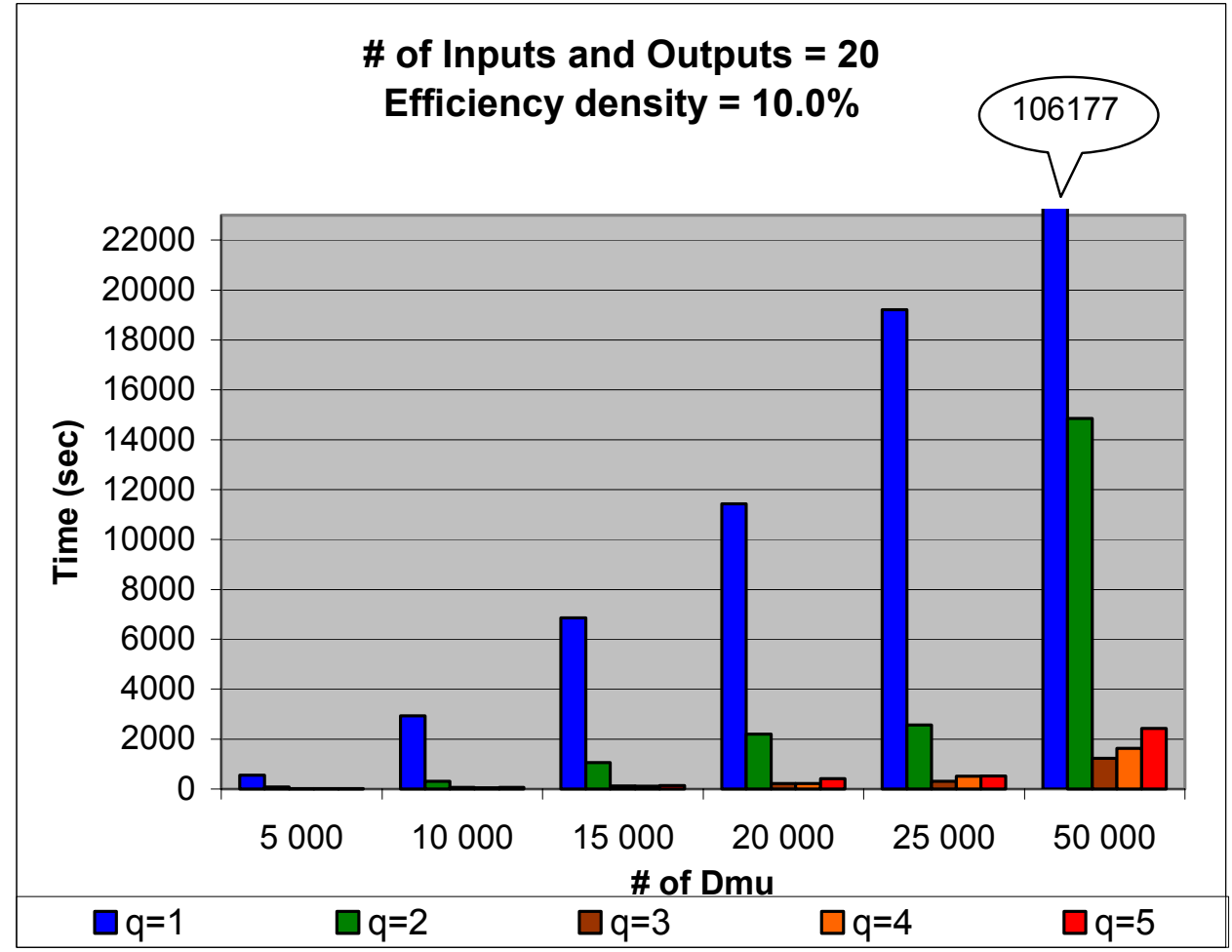

Figure 2: Computing Times (s) with Average Efficiency Density 10.0\% 


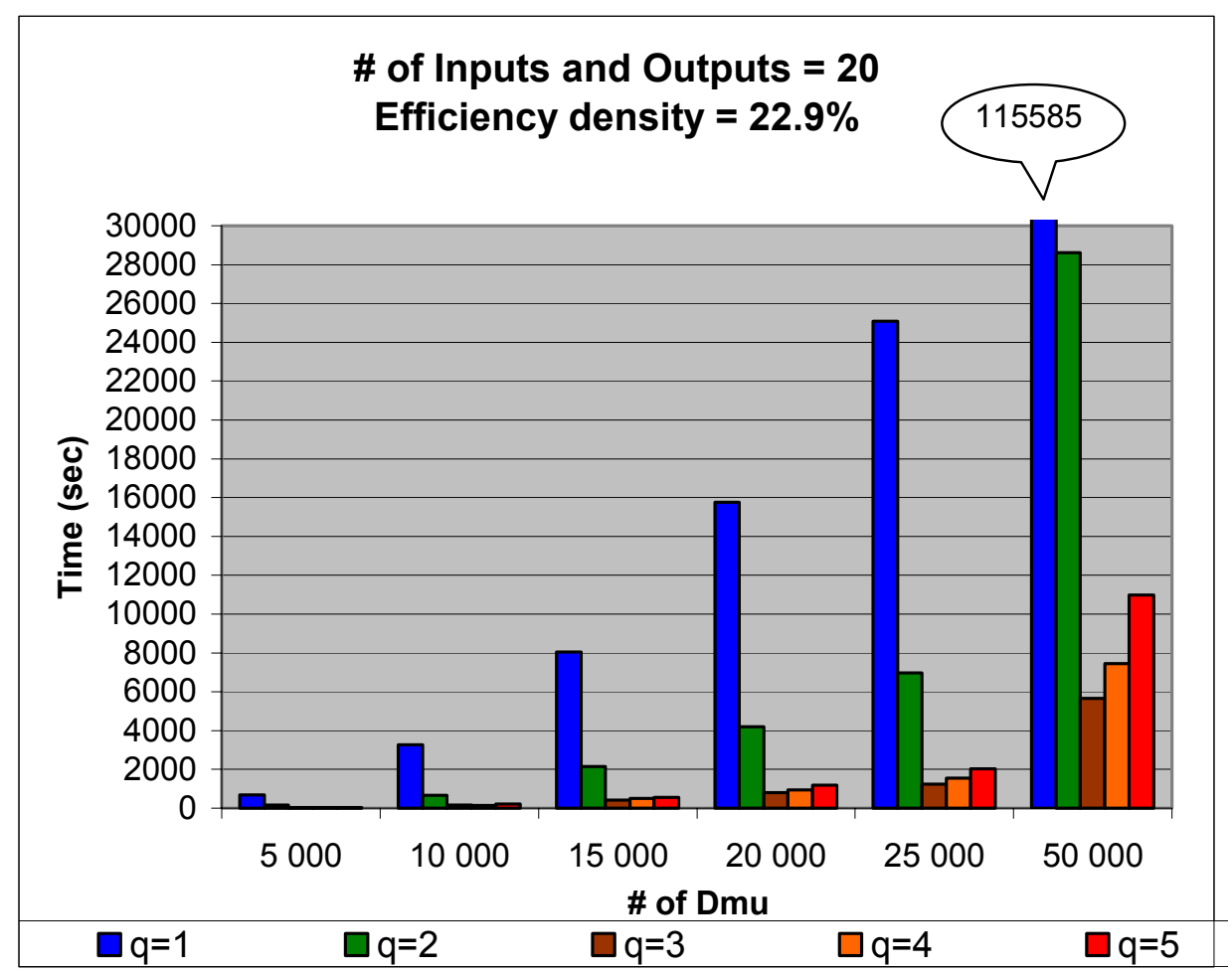

Figure 3: Computing Times (s) with Average Efficiency Density 22.9\%

The complexity of the decomposition approach depends on $n, d$, and $q$. To estimate the effect on the computing times, we have used the following regression model to fit the data in Tables 1-4:

$$
t=\alpha n^{\beta} d^{\gamma} q^{\delta} \varepsilon
$$

where $\varepsilon$ is an error term and $\alpha, \beta, \gamma$, and $\delta$ are parameters to be estimated. We took the logarithm of the model and used a linear regression to estimate the parameters. The results are represented in Table 5.

Table 5: The Estimates of the Regression Coefficients $\left(\mathrm{R}^{2}=0.918\right)$

\begin{tabular}{|l|r|r|r|r|}
\hline & Coefficients & Standard Error & Lower 95\% & Upper 95\% \\
\hline $\log (a)$ & -5.229 & 0.397 & -6.018 & -4.440 \\
\hline$b$ & 2.366 & 0.098 & 2.171 & 2.560 \\
\hline$c$ & 1.058 & 0.108 & 0.842 & 1.274 \\
\hline$d$ & -2.267 & 0.118 & -2.501 & -2.031 \\
\hline
\end{tabular}

As can be seen from Table 5, the estimate for the parameter $\delta$ is distinctly negative. This parameter value gives us a rough estimate of the magnitude of the time savings using the dimensional decomposition approach. However, it should be emphasized 
that the relationship between the logarithm of the computation time and the logarithm of the block-quantity is not linear.

\section{CONCLUSIONS}

We have developed a dimensional decomposition procedure to reduce computation times when identifying efficient and inefficient units in the Data Envelopment Analysis (DEA). Usually the algorithms developed for large-scale problems are based on the idea to decompose the problem by units. First the efficient units are recognized in sub-problems and then those units are used in computing the efficiency scores for the inefficient units.

The algorithm developed in this paper divides the problem dimensionally by selecting a subset of variables into new sub-problems. The efficient units found in sub-problems are the weakly efficient units of the original problem. They provide an initial approximation for the set of all efficient units of the original problem. The set is supplemented during the solution procedure with the units that are diagnosed efficient or super-efficient. The efficient units of the original problem are recognized from this supplemented set. The computation times are reported using five different decomposition sizes. The dimensional decomposition approach decreases computation times drastically.

In the future, our purpose is to combine the dimensional decomposition approach with the other decomposition methods that have been proposed in the literature. We also need a method which enables us to select the optimal partition sizes and the sequence of the units to be checked. These issues are topics for further research. 


\section{REFERENCES}

Afriat, S. (1972), "Efficiency Estimation of Production Functions", International Economic Review, 568-598

Andersen, P., and Petersen, N. C. (1993), "A Procedure fo Ranking Efficient Units in Data Envelopment Analysis", Management Science 39, 1261-1264.

Ali, A.I. (1993), "Streamlined Computation for Data Envelopment Analysis",

European Journal of Operational Research 64, 61-67.

Ali, A.I (1994) "Computational aspects of Data Envelopment Analysis", in Charnes, A., Cooper, W., Lewin, A.Y. and Seiford, L.M., (Eds.): Data Envelopment Analysis: Theory, Methodology, and Applications, Kluwer Academic Publishers, Norwell, 63 88.

Banker, R.D., Charnes, A. and Cooper, W.W. (1984), "Some Models for Estimating Technical and Scale Inefficiencies in Data Envelopment Analysis", Management Science 30, 1078-1092.

Barr, R.S. and Durchholz, M.L. (1997). "Parallel and Hierarchical Decomposition Approaches for Solving Large-Scale Data Envelopment Analysis Models", Annals of Operations Research 73, 339-372.

Chambers R. G., Y. Chung, and R. Färe (1998), "Profit, Directional Distance Functions, and Nerlovian Efficiency", Journal of Optimization Theory and Applications 98, 351-364.

Charnes, A., Cooper, W.W. and Rhodes, E. (1978), "Measuring Efficiency of Decision Making Units", European Journal of Operational Research 2, 429-444.

Charnes, A., Cooper, W.W. and Rhodes, E. (1979), "Short Communication: Measuring Efficiency of Decision Making Units", European Journal of Operational Research 3, 339.

Charnes, A., Cooper, W., Lewin, A.Y. and Seiford, L.M. (1994), Data Envelopment Analysis: Theory, Methodology and Applications, Kluwer Academic Publishers, Norwell.

Dulá, J.H., and Helgason, R.V. (1996), "A New Procedure for Identifying the Frame of the Convex Hull of a Finite Collection of Points in Multidimensional Space", European Journal of Operational Research 92, 352-367.

Dulá, J.H., Helgason, R.V., and Venugopal, N. (1997). "An Algorithm for Identifying the Frame of a Pointed Finite Conical Hull”, Journal of Computing 10, 323-330.

Dulá, J. H., and López, F. J. (2002), "Data Envelopment Analysis (DEA) in Massive Data Sets", in Abello, J. , Pardalos, P., and Resende, M. (Eds.): Handbook of Massive Data Sets, Kluwer Academic Publisher, pp. 419-437.

Färe, R., S. Grosskopf, and J. Logan (1983), “The Relative Efficiency of Illinois Electric Utilities", Resources und Energy 5, 349-367. 
Halme, M., Joro, T., Korhonen, P., Salo, S. and Wallenius J. (1999), "A Value Efficiency Approach to Incorporating Preference Information in Data Envelopment Analysis", Management Science 45, pp. 103-115.

Korhonen, P. and Halme, M. (1996), "Using Lexicographic Parametric Programming for Searching a Nondominated Set in Multiple Objective Linear Programming", Journal of Multi-Criteria Decision Analysis 5, 291-300.

Korhonen, P. and Siitari, P. - A. (2007): "Using Lexicographic Parametric Programming for Identifying Efficient Units in DEA", Computers \& Operations Research 34, pp. 2177-2190. 